\title{
MEDIDA INDIRETA DA PRESSÃO ARTERIAL SISTÊMICA
}

\author{
BLOOD PRESSURE MEASUREMENT
}

André Schmidt; Antônio Pazin Filho \& Benedito Carlos Maciel

\begin{abstract}
${ }^{1}$ Docentes. Disciplina de Cardiologia. Departamento de Clinica Médica da Faculdade de Medicina de Ribeirão Preto da USP. Correspondência: Prof.Dr. André Schmidt. Departamento de Clínica Médica. Faculdade de Medicina de Ribeirão Preto - USP. Campus Universitário - CEP 14048-900 - Ribeirão Preto - SP.
\end{abstract}

SCHMIDT A; PAZIN FILHO A \& MACIEL BC. Medida indireta da pressão arterial sistêmica. Medicina, Ribeirão Preto, 37: 240-245, jul./dez. 2004.

RESUMO: Este artigo revisa a medição da pressão arterial através do método indireto. São discutidos a técnica de medida e fatores que interferem no método, situações especiais e os valores de referência com base nos consensos recentemente publicados.

UNITERMOS: Pressão Arterial; medição. Semiologia.

\section{1- INTRODUÇÃO}

A medida da pressão arterial sistêmica é um procedimento fundamental na avaliação semiológica do aparelho cardiovascular. Não obstante a adequada quantificação dessa variável hemodinâmica possa ser obtida, no contexto clínico, mediante a utilização de técnicas relativamente simples, em face das importantes implicações diagnósticas e prognósticas da medida, deve-se analisar, criteriosamente, todos os fatores que podem influenciar sua aplicação. O componente mais importante do seu impacto clínico relaciona-se ao diagnóstico da Hipertensão Arterial Sistêmica (HAS). Por outro lado, do ponto de vista epidemiológico, a presença de níveis mais elevados de pressão arterial, isoladamente, modifica o prognóstico dos pacientes hipertensos no que tange à ocorrência de eventos cardiovasculares sérios, como infarto agudo do miocárdio e acidentes vasculares cerebrais. Sendo assim, uma medida inadequadamente realizada pode implicar em diagnóstico errado, determinando, conseqüentemente, a instituição de um tratamento clínico inapropriado, além da estigmatização do indivíduo, ou, ainda, determinando o oposto, como o não tratamento de paciente com indicação terapêutica.

\section{2- MÉTODOS DE MEDIDA DA PRESSÃO ARTERIAL SISTÊMICA}

A medida da pressão arterial sistêmica pode ser realizada por método direto ou indireto. A medida direta da pressão arterial é obtida de forma invasiva, mediante a introdução de um cateter em artéria periférica, o que permite sua quantificação continuamente, batimento a batimento. Tal técnica foi a primeira utilizada, pelo Reverendo Stephen Hales, no século XVIII, para medida da pressão arterial em um cavalo. No contexto clínico, a medida direta da pressão arterial é reservada para situações em que essa variável apresenta valores muito baixos, como ocorre, por exemplo, nos estados de choque circulatório.

A medida indireta da pressão arterial pode ser efetuada, utilizando-se diversas técnicas, sendo aquela realizada com o esfigmomanômetro de coluna de mercúrio ou aneróide, a mais utilizada na prática clínica diária. Exatamente por isso, o presente artigo se 
restringirá à análise desse método. O equipamento foi desenvolvido, inicialmente, pelo médico italiano Riva Rocci, em 1896, e aperfeiçoado, no que tange ao método de medida, por Nikolas Korotkoff, no início do século XX. A padronização da técnica de medida, então estabelecida, permanece praticamente inalterada desde então. Este autor verificou que, ao desinflar o manguito que ocluía totalmente a artéria, diferentes tipos de sons eram perceptíveis com o estetoscópio, o que correspondia a diferentes graus de obstrução parcial da artéria.

Os ruídos ou fases de Korotkoff podem ser detectados na maioria dos indivíduos se o procedimento de medida for executado dentro do rigor estabelecido para tal técnica. São cinco as fases:

Fase I - Corresponde ao aparecimento do primeiro som, ao qual se seguem batidas progressivamente mais fortes, bem distintas e de alta freqüência. Correlaciona-se com o nível da pressão sistólica.

Fase II - Neste momento, o som adquire característica de zumbido e sopro, podendo ocorrer sons de baixa freqüência, que eventualmente determinam o hiato auscultatório.

Fase III - Sons nítidos e intensos.

Fase IV - Abafamento dos sons, correspondendo ao momento próximo ao desaparecimento deles.

Fase V - Desaparecimento total dos sons. Correlaciona-se com a pressão diastólica.

\section{3- FATORES QUE INFLUENCIAM A MEDIDA DA PRESSÃO ARTERIAL SISTÊMICA}

A pressão arterial sistêmica é influenciada por um conjunto de fatores, que podem determinar variações significativas de seus valores ao longo do dia. A variabilidade pode ser evidenciada com a utilização da Monitoração Ambulatorial da Pressão Arterial (MAPA). Em um indivíduo normal, a pressão arterial, geralmente, sofre redução em cerca de $10 \%$, quando comparadas as medidas dos períodos de vigília e sono, o mesmo podendo ocorrer no paciente hipertenso, por vezes determinando níveis pressóricos normais durante o sono.

Entre os fatores que podem influenciar a medida da pressão arterial incluem-se aqueles relativos ao ambiente, ao equipamento, ao observador e ao paciente. $\mathrm{O}$ ambiente adequado à medida da pressão arterial deve ser tranqüilo, silencioso e com temperatura agradável. A detecção de níveis elevados de pressão arterial, em situações não ideais de medida, com ex- ceção daquelas relacionadas a emergências hipertensivas, deve ser confirmada por medidas posteriores, realizadas em local adequado.

O esfigmomanômetro, seja aneróide ou de coluna de mercúrio, deve estar adequadamente calibrado. De maneira geral, os aneróides devem ser calibrados semestralmente, enquanto que os de coluna de mercúrio, anualmente. O tamanho do manguito é de vital importância na qualidade e validade do método. Deve, nos adultos, envolver, pelo menos, $80 \%$ da circunferência braquial. Além disso, sua largura deve cobrir, pelo menos, $40 \%$ do braço. Em crianças, o manguito deve envolver $100 \%$ do braço e sua largura atingir $75 \%$ da distância entre o acrômio e o cotovelo. Portanto, deve-se medir a circunferência e tamanho do braço com fita métrica e selecionar o manguito, conforme Tabela I. Deve-se, ainda, evitar que o esfigmomanômetro e o estetoscópio estejam muito frios, $\mathrm{o}$ que pode estimular variações nos níveis de pressão.

O observador que realiza a medida da pressão arterial deve conhecer a técnica e explicá-la sucintamente ao paciente. Sua posição deve ser confortável e permitir, em caso de utilização de aparelho de coluna de mercúrio, boa visibilidade do nível da coluna. Dessa forma, serão evitadas leituras errôneas em virtude de posicionamento oblíquo em relação a ela. Em caso da utilização de aparelho aneróide, este deverá estar sob visão direta e próxima, para permitir uma distinção adequada da escala. Outra imprecisão muito freqüente na determinação da pressão arterial sistêmica é a aproximação para valores médios, terminados em zero ou cinco. Por exemplo, pressões arteriais de $98 \mathrm{mmHg}$ são aproximadas para 95 ou $100 \mathrm{mmHg}$. Além disso, por vezes, expressa-se a pressão arterial em números ímpares, apesar de a gradação da coluna de mercúrio ou aparelho aneróide apresentar escala de números pares. Considera-se isso, também, um erro sistemático, que deve ser evitado. Números ímpares podem aparecer eventualmente, quando expressarem a média de várias medições.

O paciente deve estar, também, em posição confortável, e permanecer em repouso por 5 a $10 \mathrm{mi}-$ nutos antes do início do procedimento. Ao realizar a medição na posição sentada, o tronco deve estar encostado e os braços relaxados. É ainda desejável que as pernas não estejam cruzadas. Em avaliações de consultório, deve-se esvaziar a bexiga urinária antes do procedimento e abster-se do fumo e ingestão de estimulantes (café, chá, chocolate, etc.) por, pelo menos, 30 minutos antes da medida. 


\section{4- TÉCNICA DE MEDIDA DA PRESSÃO AR- TERIAL}

A medida da pressão arterial tem sua técnica padronizada e publicada em diversas diretrizes internacionais. No Brasil, foi publicada, mais recentemente, como parte do III Consenso Brasileiro de Hipertensão Arterial. Abaixo está transcrita a seqüência de passos a serem executados para uma medida adequada da pressão arterial.

1) Explicar o procedimento ao paciente.

2) Certificar-se de que o paciente:

- não está com a bexiga cheia;

- não praticou exercícios físicos;

- não ingeriu bebidas alcoólicas, café, alimentos, ou fumou até $30 \mathrm{~min}$ antes da medida.

3) Deixar o paciente descansar por 5 a $10 \mathrm{~min}$ em ambiente calmo, com temperatura agradável.

4) Localizar a artéria braquial por palpação.

5) Colocar o manguito firmemente cerca de $2 \mathrm{~cm}$ a 3 $\mathrm{cm}$ acima da fossa antecubital, centralizando a bolsa de borracha sobre a artéria braquial. A largura da bolsa de borracha do manguito deve corresponder a $40 \%$ da circunferência do braço, e seu comprimento envolver, pelo menos, $80 \%$ do braço. Assim, a largura do manguito a ser utilizado estará na dependência da circunferência do braço do paciente (Tabela I).

6) Manter o braço do paciente na altura do coração.

7) Posicionar os olhos no mesmo nível da coluna de mercúrio ou do mostrador do manômetro aneróide.

8) Palpar o pulso radial e inflar o manguito até seu desaparecimento, para a estimativa do nível da pressão sistólica, desinflar rapidamente e aguardar de 15 a $30 \mathrm{seg}$ antes de inflar novamente.

9) Colocar o estetoscópio nos ouvidos, com a curvatura voltada para a frente.

10) Posicionar a campânula do estetoscópio, suavemente, sobre a artéria braquial, na fossa antecubital, evitando compressão excessiva.

11) Solicitar ao paciente que não fale durante o procedimento de medição.

12) Inflar rapidamente, de $10 \mathrm{mmHg}$ em $10 \mathrm{mmHg}$, até 10 a $20 \mathrm{mmHg}$ acima do nível estimado da pressão arterial.
13) Proceder à deflação, com velocidade constante inicial de $2 \mathrm{mmHg}$ a $4 \mathrm{mmHg}$ por segundo, evitando congestão venosa e desconforto para o paciente.

14) Determinar a pressão sistólica no momento do aparecimento do primeiro som (fase I de Korotkoff), que se intensifica com o aumento da velocidade de deflação.

15) Determinar a pressão diastólica no desaparecimento do som (fase V de Korotkoff), exceto em condições especiais. Auscultar cerca de $20 \mathrm{mmHg}$ a $30 \mathrm{mmHg}$ abaixo do último som, para confirmar seu desaparecimento e, depois, proceder à deflação rápida e completa. Quando os batimentos persistirem até o nível zero, determinar a pressão diastólica no abafamento dos sons (fase IV de Korotkoff) e anotar o valor final de zero, por exemplo, 136/78/0.

16) Registrar os valores das pressões sistólica e diastólica, complementando com a posição do paciente, o tamanho do manguito e o braço em que foi feita a mensuração. Deverá ser registrado sempre o valor da pressão, obtido na escala do manômetro, que varia de $2 \mathrm{mmHg} \mathrm{em} 2 \mathrm{mmHg}$, evitando-se arredondamentos e valores de pressão terminados em "5".

17) Esperar 1 a 2 min antes de realizar novas medidas.

18) O paciente deve ser informado sobre os valores da pressão arterial e a possível necessidade de acompanhamento.

Em caso de dificuldade para auscultar os ruídos de Korotkoff, pode-se lançar mão de uma manobra simples: fechar e abrir a mão do membro em que a pressão está sendo medida, durante alguns segundos.

\begin{tabular}{|c|l|c|c|}
\hline \multicolumn{2}{|c|}{ Tabela I: Dimensões recomendadas da bolsa inflável do manguito. } \\
\hline $\begin{array}{c}\text { Circunferência } \\
\text { do braço (cm) }\end{array}$ & $\begin{array}{c}\text { Denominação do } \\
\text { manguito }\end{array}$ & $\begin{array}{c}\text { Largura da } \\
\text { bolsa }(\mathbf{c m})\end{array}$ & $\begin{array}{c}\text { Comprimento } \\
\text { da bolsa (cm) }\end{array}$ \\
\hline $5-7,5$ & Recém-nascido & 3 & 5 \\
\hline $7,5-13$ & Lactente & 5 & 8 \\
\hline $13-20$ & Criança & 8 & 13 \\
\hline $17-24$ & Adulto magro & 11 & 17 \\
\hline $24-32$ & Adulto & 13 & 24 \\
\hline $32-42$ & Adulto obeso & 17 & 32 \\
\hline $42-50$ & Coxa & 20 & 42 \\
\hline
\end{tabular}


A etapa de avaliação da Pressão Sistólica Estimada (PSE) é importante para se evitar uma série de inconvenientes e erros na medida indireta da pressão arterial. Em primeiro lugar, ao estimar a pressão sistólica pela técnica de palpação, evitando-se insuflações exageradas, causadoras de desconforto para o paciente e erro na medição. Em segundo lugar, evitam-se erros decorrentes do fenômeno conhecido como "hiato auscultatório". Este evento decorre de curto intervalo, em que os ruídos de Korotkoff não são audíveis, e pode estender-se por intervalos de até $40 \mathrm{mmHg}$. Ao realizar a medida da pressão arterial sem utilizar a PSE, pode-se interromper, eventualmente, a insuflação dentro do período de hiato auscultatório, e, desse modo, obter leituras falsamente baixas para a pressão sistólica. Esse fenômeno ocorre, geralmente, em idosos com HAS, arteriosclerose e estenose aórtica grave. Deve-se, também, evitar insuflações e deflações repetidas do manguito durante uma mesma medida, o que pode provocar estímulos dolorosos e variação dos valores da pressão arterial.

\section{5- SITUAÇÕES ESPECIAIS DE MEDIDA DA PRESSÃO ARTERIAL}

A medida da pressão arterial, nos membros inferiores, pode ser realizada sempre que houver impossibilidade nos membros superiores ou em caso de suspeita de doença vascular. Neste caso, deve ser utilizado manguito mais largo, que respeite as relações acima descritas. O paciente deve, preferencialmente, posicionar-se em decúbito ventral, com o manguito acoplado ao terço inferior da coxa e o estetoscópio sobre a artéria poplítea. É importante ressaltar que, em condições normais, a pressão sistólica é 20 a 30 $\mathrm{mmHg}$ mais elevada nos membros inferiores, em relação aos membros superiores, enquanto a pressão diastólica é semelhante. Na presença de algumas doenças vasculares (Coarctação de Aorta ou obstruções vasculares), a pressão sistólica apresenta valores menores que aqueles observados nos membros superiores.

Em estados de choque circulatório, pode ser impossível caracterizar os ruídos de Korotkoff, e a PSE pode ser a única técnica indireta para estimativa da pressão sistólica. Nesses casos, geralmente se recorre à medida direta da pressão arterial.

Pacientes com suspeita de hipotensão ortostática devem ter a pressão arterial medida na posição ortostática. Em tal caso, deve-se tomar o cuidado de posicionar o braço ao qual o manguito está acoplado na altura do coração, recorrendo a suportes fixos, ou com o auxílio de terceiros. Os valores de redução são, muitas vezes, arbitrários, devendo-se sempre correlacionar a queda nos níveis de pressão de pelo menos $20 \mathrm{mmHg}$ com a história clínica de sintomas correspondentes. A medida deve ser feita após o paciente permanecer deitado por cinco minutos, pelo menos, medindo-se imediatamente após levantar-se e a cada 2 min, durante até $10 \mathrm{~min}$, em casos muito suspeitos. Não se deve utilizar medidas de pressão com o paciente sentado.

Pulso paradoxal é definido como redução superior a dez milímetros de mercúrio da pressão arterial sistólica durante a inspiração. Apesar de classicamente associado ao tamponamento cardíaco, pode ocorrer também em situações clínicas, como doença pulmonar obstrutiva crônica, insuficiência respiratória aguda e asma brônquica.

A determinação do pulso paradoxal requer técnica adequada. Inicialmente, insufla-se o manguito cerca de $10 \mathrm{mmHg}$ acima do ponto em que desaparece o pulso braquial. Em seguida, coloca-se o estetoscópio sobre a artéria braquial e inicia-se a desinsuflação do manguito de modo gradual até que se ausculte o primeiro ruído de Koratkoff. Neste ponto, oclui-se o manguito e observa-se a respiração do paciente. Se o ruído desaparecer com a inspiração, constata-se que a pressão arterial sistólica está caindo com a inspiração. Prossegue-se com a desinsuflação do manguito para $5 \mathrm{mmHg}$ abaixo desse ponto inicial e novamente observa-se a inspiração; se o ruído desaparecer, nesse ponto, constata-se que a queda da pressão arterial sistólica é de pelo menos $5 \mathrm{mmHg}$. Repete-se esta etapa, com reduções sucessivas de $5 \mathrm{mmHg}$, até que não se perceba mais o desaparecimento do ruído com a inspiração. Quando isto ocorrer, determina-se o valor de queda como sendo o total de etapas em que esta queda foi documentada, multiplicado por $5 \mathrm{mmHg}$.

Em caso de suspeita de processos obstrutivos arteriais acometendo a aorta e seus ramos torácicos ou de membros superiores, a medida da pressão arterial pode apresentar diferenças de 20 ou mais $\mathrm{mmHg}$ na pressão sistólica.

Em situações clínicas especiais, em que se documenta redução da resistência vascular periférica, a pressão diastólica é melhor determinada pelo abafamento dos sons de Korotkoff e não ao seu desaparecimento. Isso pode ocorrer na insuficiência aórtica.

Finalmente, o diagnóstico de pseudo-hipertensão, presente em muitos pacientes idosos, pode ser esclarecido com manobras simples. Esse diagnóstico decorre da rigidez das artérias, com calcificação, con- 
seqüente à idade avançada. No caso, ao inflar o manguito, podemos encontrar valores falsamente elevados de pressão arterial, em decorrência da dificuldade para ocluir a artéria braquial. Então, podemos recorrer à manobra de Osler. Tal manobra consiste em inflar o manguito até que ultrapasse a pressão sistólica. Caso a artéria braquial ou radial do membro em que está sendo insuflado o manguito permaneça palpável, considera-se "Osler positivo". Se não for mais palpada, significa que colapsou e, portanto, 'considera-se "Osler negativo". A positividade da manobra indica que o vaso é rígido e existe a possibilidade de estarmos diante de um caso de pseudo-hipertensão. Contudo, resposta definitiva só poderá ser obtida com a medida direta da pressão arterial. Em tais casos, não se exclui o diagnóstico de HAS, mas, eventualmente, os níveis tensionais são menores que os medidos pela técnica indireta.

\section{6- VALORES DE PRESSÃO ARTERIAL}

Na Tabela II, estão expressos os valores de pressão arterial considerados na avaliação diagnósti-
Tabela II: Classificação da Pressão Arterial, segundo o III Consenso Brasileiro de Hipertensão Arterial e VI JNC (Consenso Americano.)

\begin{tabular}{|c|c|c|c|}
\hline Categoria & $\begin{array}{r}\text { Sistólica } \\
(\mathbf{m m H g})\end{array}$ & & $\begin{array}{c}\text { Diastólica } \\
(\mathrm{mmHg})\end{array}$ \\
\hline Ótima & $<120$ & e & $<80$ \\
\hline Normal & $<130$ & e & $<85$ \\
\hline Normal Alta & $130-139$ & ou & $85-89$ \\
\hline $\begin{array}{l}\text { Hipertensão } \\
\text { - Estágio I } \\
\text { - Estágio II } \\
\text { - Estágio III }\end{array}$ & $\begin{array}{c}- \\
140-159 \\
160-179 \\
\square 180\end{array}$ & $\begin{array}{l}\text { ou } \\
\text { ou } \\
\text { ou }\end{array}$ & $\begin{array}{c}- \\
90-99 \\
100-109 \\
\square 110\end{array}$ \\
\hline
\end{tabular}

ca e prognóstica da população adulta com dezoito anos ou mais e sem doenças agudas concomitantes.

A Tabela III expressa os valores para o diagnóstico de HAS em crianças e adolescentes. Nesse caso, o valor é definido com base, além da idade, no

Tabela III: Valores da Pressão Arterial em crianças e adolescentes, expressos segundo percentil de altura e sexo.

\begin{tabular}{|c|c|c|c|c|c|c|}
\hline \multirow[t]{2}{*}{$\begin{array}{l}\text { Idade } \\
\text { (anos) }\end{array}$} & \multirow[t]{2}{*}{$\begin{array}{c}\text { Estatura } \\
\text { Percentil }(\mathrm{cm})\end{array}$} & \multicolumn{2}{|c|}{$\begin{array}{l}\text { Sexo Masculino } \\
\text { Pressão Arterial } \\
\text { (mmHg)- Percentil }\end{array}$} & \multirow[t]{2}{*}{$\begin{array}{c}\text { Estatura } \\
\text { Percentil }(\mathrm{cm})\end{array}$} & \multicolumn{2}{|c|}{$\begin{array}{l}\text { Sexo Feminino } \\
\text { Pressão arterial } \\
\text { (mmHg) Percentil }\end{array}$} \\
\hline & & P 90 & P 95 & & P 90 & P 95 \\
\hline 1 & $\begin{array}{l}50 \%(76) \\
75 \%(78)\end{array}$ & $\begin{array}{l}98 / 53 \\
100 / 54\end{array}$ & $\begin{array}{l}102 / 57 \\
104 / 58\end{array}$ & $\begin{array}{l}50 \%(74) \\
75 \%(77)\end{array}$ & $\begin{array}{l}100 / 54 \\
102 / 55\end{array}$ & $\begin{array}{l}104 / 58 \\
105 / 59\end{array}$ \\
\hline 3 & $\begin{array}{l}50 \%(97) \\
75 \%(99)\end{array}$ & $\begin{array}{l}105 / 61 \\
107 / 62\end{array}$ & $\begin{array}{l}109 / 65 \\
111 / 66\end{array}$ & $\begin{array}{l}50 \%(96) \\
75 \%(98)\end{array}$ & $\begin{array}{l}103 / 62 \\
104 / 63\end{array}$ & $\begin{array}{l}107 / 66 \\
108 / 67\end{array}$ \\
\hline 6 & $\begin{array}{l}50 \%(116) \\
75 \%(119)\end{array}$ & $\begin{array}{l}110 / 70 \\
111 / 70\end{array}$ & $\begin{array}{l}114 / 74 \\
115 / 75\end{array}$ & $\begin{array}{l}50 \%(115) \\
75 \%(118)\end{array}$ & $\begin{array}{l}107 / 69 \\
109 / 69\end{array}$ & $\begin{array}{l}111 / 73 \\
112 / 73\end{array}$ \\
\hline 9 & $\begin{array}{l}50 \%(132) \\
75 \%(136)\end{array}$ & $\begin{array}{l}113 / 74 \\
115 / 75\end{array}$ & $\begin{array}{l}111 / 79 \\
119 / 80\end{array}$ & $\begin{array}{l}50 \%(132) \\
75 \%(137)\end{array}$ & $\begin{array}{l}113 / 73 \\
114 / 74\end{array}$ & $\begin{array}{l}117 / 77 \\
118 / 78\end{array}$ \\
\hline 12 & $\begin{array}{c}50 \%(150) \\
75 \% \quad(155)\end{array}$ & $\begin{array}{l}119 / 77 \\
121 / 78\end{array}$ & $\begin{array}{l}123 / 81 \\
125 / 82\end{array}$ & $\begin{array}{l}50 \%(152) \\
75 \%(155)\end{array}$ & $\begin{array}{l}119 / 76 \\
120 / 77\end{array}$ & $\begin{array}{l}123 / 80 \\
124 / 81\end{array}$ \\
\hline 15 & $\begin{array}{l}50 \%(168) \\
75 \%(174)\end{array}$ & $\begin{array}{l}127 / 79 \\
129 / 80\end{array}$ & $\begin{array}{l}131 / 83 \\
133 / 84\end{array}$ & $\begin{array}{l}50 \%(161) \\
75 \%(166)\end{array}$ & $\begin{array}{l}124 / 79 \\
125 / 80\end{array}$ & $\begin{array}{l}128 / 83 \\
129 / 84\end{array}$ \\
\hline 17 & $\begin{array}{l}50 \%(176) \\
75 \%(180)\end{array}$ & $\begin{array}{l}133 / 83 \\
134 / 84\end{array}$ & $\begin{array}{l}136 / 87 \\
138 / 88\end{array}$ & $\begin{array}{l}50 \%(163) \\
75 \%(167)\end{array}$ & $\begin{array}{l}125 / 80 \\
126 / 81\end{array}$ & $\begin{array}{l}129 / 84 \\
130 / 85\end{array}$ \\
\hline
\end{tabular}

Para efeito diagnóstico, seria considerada a seguinte classificação:- valores abaixo do percentil 90 = normotensão; - valores entre os percentis 90 e 95 = normal limítrofe; - valores acima do percentil 95 = hipertensão arteri.al. 
percentil de altura e pelo sexo da criança ou adolescente. É importante salientar a possibilidade de uma causa secundária para a HAS em tal faixa etária, abrindo perspectivas para a prevenção de lesões em órgãos-alvo.

Para efeito diagnóstico, seria considerada a seguinte classificação: valores abaixo do percentil $90=$ normotensão valores entre os percentis 90 e
95 = normal limítrofe valores acima do percentil $95=$ hipertensão arterial

Convém lembrar que o diagnóstico deve ser realizado dentro de critérios rígidos, que fogem ao escopo desta publicação, e que a medida indireta, realizada de forma criteriosa como a descrita acima, possibilitará melhor acurácia e reprodutibilidade no acompanhamento dos pacientes.

SCHMIDT A; PAZIN FILHO A \& MACIEL BC. Blood pressure measurement. Medicina, Ribeirão Preto, 37: 240-245, july/dec. 2004.

ABSTRACT: This article reviews the basis of indirect blood pressure measurement. The technical aspects, confounding factors and special situations, and the normal values supported by literature consensus are reviewed.

UNITERMS: Blood Pressure; measurement. Semiology.

\section{BIBLIOGRAFIA RECOMENDADA}

1 - III CONSENSO BRASILEIRO DE HIPERTENSÃOARTERIAL - http:/ /publicacoes.cardiol.br/consenso/

2 - LÓPEZ M \& LAURENTIS-MEDEIROS J. Semiologia médica - As bases do diagnóstico clínico. $4^{\circ}$ ed. Revinter, Rio de Janeiro, 1999.
3 - PORTO CC. Semiologia médica. $3^{\mathrm{a}}$. ed. Guanabara Koogan, Rio de Janeiro, 1997. 